\title{
Boundary Layer Transition over Blunt Hypersonic Vehicles Including Effects of Ablation-Induced Out-Gassing
}

\author{
Fei Li ${ }^{*}$, Meelan Choudhari ${ }^{\dagger}$, Chau-Lyan Chang ${ }^{\&}$, and Jeffery White ${ }^{\&}$ \\ NASA Langley Research Center, Hampton, VA 23681
}

\begin{abstract}
Computations are performed to study the boundary layer instability mechanisms pertaining to hypersonic flow over blunt capsules. For capsules with ablative heat shields, transition may be influenced both by out-gassing associated with surface pyrolysis and the resulting modification of surface geometry including the formation of micro-roughness. To isolate the effects of out-gassing, this paper examines the stability of canonical boundary layer flows over a smooth surface in the presence of gas injection into the boundary layer. For a slender cone, the effects of out-gassing on the predominantly second mode instability are found to be stabilizing. In contrast, for a blunt capsule flow dominated by first mode instability, out-gassing is shown to be destabilizing. Analogous destabilizing effects of outgassing are also noted for both stationary and traveling modes of crossflow instability over a blunt sphere-cone configuration at angle of attack.
\end{abstract}

\section{Nomenclature}



\section{Subscripts}

$\begin{array}{ll}c f & =\text { crossflow } \\ e & =\text { boundary layer edge } \\ n & =\text { wall-normal direction } \\ w & =\text { wall } \\ \infty & =\text { freestream }\end{array}$

\footnotetext{
* Aerospace Technologist, Computational AeroSciences Branch, M.S. 128.

$\dagger$ Aerospace Technologist, Computational AeroSciences Branch, M.S. 128. Associate Fellow, AIAA.

\& Aerospace Technologist, Computational AeroSciences Branch, M.S. 128. Senior Member, AIAA.
} 


\section{Introduction}

Blunt aeroshells with ablative heat shields are often used to survive the high energy entries into a planetary atmosphere. The Orion or Crew Exploration Vehicle (CEV) designed to support human space missions returning from low earth orbit and the moon will have to use an ablative heat shield at lunar reentry velocities. Whereas stability characteristics and associated transition mechanisms for smooth surface boundary layer flows over slender body vehicles are reasonably well understood at this point, those for the blunt capsule vehicles are just beginning to be addressed. ${ }^{1,2}$ Given its influence on surface heat transfer, skin friction drag, and flow separation characteristics, understanding and prediction of boundary layer transition over the vehicle surface constitutes an important aspect of hypersonic vehicle design.

A number of experiments have been performed to study the effect of surface ablation on boundary layer transition. Typically, most experiments have focused on measuring the effects of surface out-gassing ${ }^{3-5}$ alone or the role of ablation induced distributed roughness, ${ }^{6}$ but not both. Roughness induced transition in a high-speed boundary layer is a difficult problem. ${ }^{7}$ However, an intense fundamental research effort funded by NASA and AFRL over the past few years has provided significant clues into the physical mechanisms underlying transition due to discrete surface roughness (i.e. see references cited in Ref. 8). Extension of these studies to distributed surface roughness is yet to be undertaken. On the computational side, aerothermal analysis for ablative heat shields has traditionally been performed without a direct coupling between ablation and the material response. However, considerable effort has been devoted in recent years towards the eventual development of a coupled capability by implementing surface mass transfer boundary conditions into CFD solvers. ${ }^{9,10}$ The effects of surface blowing on boundary layer instability have also been studied recently in the context of a flat plate boundary layer. ${ }^{11,12}$

This paper is focused on the transition mechanisms due to the effects of out-gassing in the absence of surface roughness. Previous studies related to the effects of blowing on flat plate boundary layers ${ }^{11,12}$ are extended first to a slender cone and then to a blunt capsule. Results for each of these geometries are presented in Sections III, IV and V below, following a description of the analysis. For simplicity, the flow conditions are such that no high-enthalpy effects are expected in either of the two flow configurations.

\section{Analysis Codes}

The unperturbed boundary layer flow over the cone surface was computed on various grids using a second order accurate algorithm as implemented in a finite-volume compressible Navier-Stokes flow solver VULCAN. ${ }^{\ddagger}$ The VULCAN computations utilized the code's built-in capability to accomplish shock adaptations. The out-gassing surface boundary condition implemented in VULCAN was based on the methodology described in Ref. 10. This methodology requires the specification of the surface normal mass flux, gas composition and static temperature. A quadratic equation is solved to obtain the surface density and the surface pressure is finally computed via the ideal gas law. The VULCAN implementation also included the flexibility to specify an arbitrary out-gassing distribution (or profile) on the boundary surface. In selected cases, the mean flow was computed on multiple grids to enable an assessment of grid convergence. In select cases, the mean flow was computed on multiple grids to enable an assessment of grid convergence. For the case of a slender cone, the mean flow was also computed using a boundary layer solver ${ }^{13}$ to ensure that the effects of out-gassing on stability characteristics based on the NavierStokes mean flow compare well with the results based on mean flow obtained by solving boundary layer equations.

\footnotetext{
${ }^{*}$ http://vulcan-cfd.larc.nasa.gov
} 
The stability of the computed boundary layer flow was analyzed using the Langley Stability and Transition Analysis Code (LASTRAC). ${ }^{14}$ Analysis was performed using both quasi-parallel stability theory and the parabolized stability equations (PSE). Sutherland's law is assumed to describe the viscosity variation for both the mean flow and the unsteady perturbations associated with boundary layer instability waves. Stokes law is assumed for bulk viscosity.

\section{Slender Cone}

First, consider the effects of air injection into a Mach 7.4, air boundary layer over a 5-degree semi-angle circular cone with a sharp tip. The injection was turned on at $s=L$, where $s$ denotes the distance from the tip along a cone generator and $L \equiv 0.09525$ meters ( $3.75 \mathrm{in}$.) corresponds to the location where the surface mass injection begins in the experiment ${ }^{4}$ modeled herein. The flow conditions and the spatial distribution of injection velocity are modeled after Ref. 4 . The flow conditions are shown in Table I and perfect gas behavior is assumed.

Table I. Flow conditions for the slender body.

\begin{tabular}{|c|c|}
\hline Mach & 7.4 \\
\hline Unit Reynolds Number $(1 / \mathrm{m})$ & $9.252 \times 10^{6}$ \\
\hline Freestream Temperature $(\mathrm{K})$ & 69.72 \\
\hline Surface temperature $(\mathrm{K})$ & 308 \\
\hline Angle of Attack (deg.) & 0 \\
\hline
\end{tabular}

The computational grids used for most of the axisymmetric mean flow computations (corresponding to varying levels of surface mass flux) have 1153 points along the length of the cone and 513 points in the wall normal direction. The sharp tip of the cone is approximated by a spherical tip that is $4.572 \times 10^{-6}$ meters in radius and is smoothly joined by a $4^{\text {th }}$ order polynomial to the straight side of the cone. The spherical cone tip is resolved by approximately 40 points up to where the sphere tangentially joins the straight side. The normal grid is clustered near the wall with approximately 200 points to resolve the boundary layer thickness. The length of the cone throughout all computations is fixed at 0.508 meters. A denser grid is also constructed with double the number of grid points in each direction to verify grid convergence of the mean surface heat flux and boundary layer profiles. Tangential velocity and temperature profiles at selected locations along the cone surface are plotted in Fig. 1, which includes the results obtained using both of these grids. Virtually no difference can be discerned between the two sets of profiles. Mean flow solutions were also obtained using an alternate boundary condition corresponding to boundary layer bleed and the profiles (not shown) matched well with the solutions shown in Fig. 1.

Computations are performed for five selected cases with increasing surface mass flux but an identical mass flux distribution along the cone surface based on the measured data as reported in Ref. 4. Case I corresponds to an impermeable cone surface (i.e. zero mass injection), whereas cases II through $\mathrm{V}$ correspond to increasing surface mass flux. The surface mass flux distribution for case II is shown in Fig.2. The "amplitude" of the surface mass flux for cases I (zero mass flow), IV and V was selected to provide a close match between computed and measured surface heating distribution (Fig. 3).

Heat flux distributions for five selected magnitudes of the overall surface mass flux are plotted in Fig. 3, including a comparison with heat flux data from the experiment. It is seen that, for cases I, IV, and V, the surface heat fluxes match those in the experiment. Because of certain ambiguities with the actual mass 
flux distribution in the experiment, the "amplitude" of the surface mass flux in the computations was selected to provide a close match with the surface heating distribution.

In high Mach number flows, the dominant instability mechanism is associated with a generalized inflection point in the boundary layer profile, which is defined as the location where the product of density and the normal gradient of streamwise velocity has a local maximum. Fig. 4 shows wall-normal profiles of this product at various streamwise locations for cases I, IV and V. The peaks in these profiles are clearly seen. For case I, namely, the zero mass flux case, the boundary layer is approximately selfsimilar and, therefore, all profiles nearly collapse onto one another. With finite surface mass flux for cases IV and V, the boundary layer thickens and the generalized inflection point moves away from the wall.

Amplification characteristics of second mode waves for cases I, IV, and V, respectively, are computed for a range of frequencies from 100 to $775 \mathrm{kHz}$ in $25 \mathrm{kHz}$ intervals using the linearized version of parabolized stability equations (PSE). The logarithmic amplification ratio ( $\mathrm{N}$-factor) for axisymmetric second mode disturbances at selected frequencies is plotted in Fig. 5 as a function of the streamwise coordinate normalized by $L$, the distance from the cone tip at which the surface mass flux is switched on. Results in Fig. 5(a) correspond to the baseline case of an impermeable cone, whereas those in Fig. 5(b) and 5(c) correspond to case IV and case V, respectively, from Fig. 3. It is interesting to observe that the peak $\mathrm{N}$-factors in Fig. 5(b) and 5(c) are actually lower than that in Fig. 5(a), indicating that the second mode disturbances are stabilized by the presence of mass injection in this case. Furthermore, the peak frequency is reduced from $250 \mathrm{kHz}$ in case I to $175 \mathrm{kHz}$ in case $\mathrm{V}$, reflecting the effect of boundary layer thickening. The physical mechanism behind the stabilizing effect of out-gassing on the second mode disturbances has not been identified using current computations. However, one interesting feature of the amplification characteristics at nonzero injection corresponds to the extended region of nearly constant $\mathrm{N}$ factor at large $\mathrm{x}$ (see, for instance, the $\mathrm{N}$-factor curve for the most amplified disturbance of $250 \mathrm{kHz}$ in Fig. 5(b), which exhibits an alternating weak decay and weak growth at $x / L>3.5$ ).

The above results show a stabilizing effect of out-gassing on second mode amplification. The experiment, on the other hand, indicated a forward movement in transition with an increasing magnitude of the blowing velocity at the surface. This can only mean that the earlier transition in the experiment could not have been caused by the second-mode instability mechanism. The question of what exactly led to the earlier transition in the experiment remains to be ascertained, especially since it appears unlikely that the injection mechanism also acted as a source of unsteady disturbances in the range of second mode frequencies, contributing to a significant increase in receptivity for the nonzero injection cases. One other possibility that we are investigating at present corresponds to the effect of the porous surface (which is not modeled in our current stability equations) on disturbance growth at the frequencies of interest.

\section{Blunt Capsule}

Findings for a Mach 7.32 flow over a blunt, hemi-spherical capsule are presented next, where, again, we assume the injection species to be air, i.e., the same as the incoming flow. The radius of the body is 0.0889 meters $(3.5$ in). The flow conditions are listed in Table II. The Mach number at the boundary layer edge is subsonic over a significant portion of the body length and the maximum edge Mach numbers have low supersonic values. Mach number contours for the baseline case (zero blowing) and the case of maximum injection velocity used this study (4 percent blowing) are shown in Figs. 6(a) and 6(b), respectively. Besides its obvious impact in substantial thickening of the boundary layer region near the surface, the out-gassing also influences the shock location, which gets pushed successively further from the body albeit by a small distance. 
Table II. Flow conditions for a Mach 7.32 hemi-spherical capsule.

\begin{tabular}{|c|c|}
\hline Mach Number & 7.32 \\
\hline Unit Reynolds Number $(1 / \mathrm{m})$ & $14.6 \times 10^{6}$ \\
\hline Freestream Temperature $(\mathrm{K})$ & 65 \\
\hline Surface Temperature $(\mathrm{K})$ & 300 \\
\hline Angle of Attack (deg.) & 0 \\
\hline
\end{tabular}

The computational accuracy was assessed by using three grids belonging to the same family, but with significantly different sizes. An example of the grid convergence test for an injection mass flux of $4 \%$ is shown in Fig. 7. The coarsest grid has 129 points along the hemi-sphere surface and 353 points in the wall normal direction with at least 100 points in the boundary layer. The two finer grids have, respectively, twice and four times the grid points in each direction. Comparison of boundary layer profiles based on these grids shows that the coarsest grid has slight inaccuracies in this high injection case, while the two finer grids yield grid-converged solutions except in the region very close to the outflow boundary (Fig. 7). For stability and transition analysis with region of interest far ahead of the outflow boundary, the midlevel grid is, therefore, sufficient to ensure accurate computations of the growth of instability waves.

For the zero mass injection case, the boundary layer flow over the hemispherical capsule does not support any significant amplification of instability modes. The absence of instability in the baseline case is attributed to the strong favorable pressure gradient over the body surface coupled with a sufficiently low Reynolds number. The boundary layer profiles do not have any generalized inflection point, which, as explained in Section III, is defined as the location where the product of density and the normal gradient of streamwise velocity has a local maximum. Hence, it cannot support any instability modes associated with an inviscid mechanism.

Introduction of mass transfer at the surface produces a dramatic effect on the flow stability when the injection rate parameter, $\mathrm{m}$, becomes sufficiently large. Here, $m \equiv \rho_{w} V_{w} / \rho_{\infty} U_{\infty}$, where $\rho$ denotes the fluid density, $V$ represents the velocity normal to the wall, subscripts $w$ and $\infty$ denote conditions at the wall and in free-stream, respectively. At $m=0.01$, for example, the previously non-inflectional boundary layer profiles have developed a very pronounced peak in the middle of the boundary layer, which moves farther away from the surface (both in absolute sense and relative to the boundary layer thickness) at increasing distance from the stagnation point (Fig. 8). This modification of mean flow profiles results in the onset of inflectional first mode instability. Representative mode shapes associated with the streamwise velocity fluctuation produced by this instability are shown in Fig. 9. Consistent with the outward movement in inflection point location at increasing distance from the stagnation point, the peak of the (normalized) $u$ mode shape moves farther away from the surface as the distance parameter $X$ is increased. Here, $u$ denotes the streamwise (i.e., tangential to the surface) velocity fluctuation associated with the instability wave.

The most amplified first mode instability is, in general, an oblique wave. This means that a very large parameter range in the frequency and azimuthal wave number space need to be searched to find the maximum amplification. However, in this particular case, the boundary-layer edge flow over most of the region of interest is subsonic and test runs at a few representative locations on the surface confirm that the most amplified first mode waves for all relevant frequencies are very nearly (if not exactly) axisymmetric ( i.e. having zero azimuthal wavenumber). Therefore, in the first mode instability analysis below, only axisymmetric first mode waves are sought, which reduces the parameter range of search by an order of magnitude. 
The effect of out-gassing on the amplification of these inflectional instabilities is shown in Fig. 10, where cumulative amplification ratios ( $\mathrm{N}$-factors) for selected fixed-frequency disturbances are plotted for two values of the out-gassing parameters, $m=0.01$ and $m=0.013$. All $\mathrm{N}$-factors are computed with the quasiparallel assumption with streamwise surface curvature effects accounted for. Despite the fact that the injection velocity increases by only 30 percent from Fig. 10(a) to Fig. 10(b), the associated value of peak $\mathrm{N}$-factor is nearly doubled. This suggests that the transition onset location will rapidly shift inward once the out-gassing parameter has crossed some threshold value. These observations are qualitatively consistent with the measurements by Kaattari. ${ }^{3}$ A quantitative comparison with the measured transition locations will require using the non-uniform mass flux distribution inferred from the measurements, along with an analysis of the impact of the associated uncertainties on the instability wave amplification. These computations are currently under way and will be reported in a future paper.

\section{Mars Science Lab (MSL) Capsule}

To examine the effect of surface injection on crossflow instabilities on a blunt configuration, a spherecone configuration that was tested in the wind tunnel for the Mars Science Laboratory (MSL) is considered. ${ }^{15}$ The model has a spherical nose of radius $38.1 \mathrm{~mm}$, which smoothly joins a straight side of 50 degree semi-cone angle. The base radius of the model is $80 \mathrm{~mm}$. The flow conditions are listed in Table III. Again, the perfect gas assumption is used and the mass-flux distribution along the front surface is assumed to be uniform except in the immediate vicinity of the shoulder region adjacent to the base of the model, where it is tapered to zero.

Table III. Flow conditions for a Mach 6 MSL capsule model.

\begin{tabular}{|c|c|}
\hline Mach Number & 6.0 \\
\hline Unit Reynolds Number $(1 / \mathrm{m})$ & $15 \times 10^{6}$ \\
\hline Freestream Temperature $(\mathrm{K})$ & 60 \\
\hline Surface Temperature $(\mathrm{K})$ & 300 \\
\hline Angle of Attack (deg.) & 16 \\
\hline
\end{tabular}

Two grids belonging to the same family, but of different sizes, are used to verify the grid convergence of the mean boundary layer flow over the 50-deg sphere cone configuration. The coarse grid has $97 \times 65 \times 353$ points in the streamwise, azimuthal and wall-normal direction, respectively, whereas the denser grid has 1.5 times the number of grid points in the streamwise and azimuthal directions, respectively (145x97x353). Fig.11(a) and 11(b) show the boundary layer velocity profiles along the windward and leeward symmetry lines. Slight differences are observable at large distance away from the nose of the model, but they are not expected to affect the stability analysis within the main region of interest.

Figs. 12(a) and 12(b) show, respectively, the mean flow density contours on the model wall and the Mach contours in the plane of symmetry that contains the windward and leeward lines. The Mach number contours indicate the shift in the stagnation point to the lower half of the model. The flow within the shock layer region on the lower, windward side corresponds to lower Mach numbers but higher temperatures in comparison with the flow on the leeward side.

One of the important gauges of crossflow instability is the crossflow Reynolds number. The effect of outgassing on crossflow Reynolds number is shown in Fig. 13, demonstrating a significant increase in the maximum crossflow Reynolds number as a result of out-gassing, specifically from about 640 to approximately 750 .

To further assess the grid convergence for nonzero out-gassing, stability computations were performed with the above two grids for the stationary crossflow instability along a streamline that cuts through the 
region of peak crossflow Reynolds numbers (viz., the $4^{\text {th }}$ streamline from the leeward symmetry plane in Fig. 14 below). The maximum stationary crossflow $\mathrm{N}$ factor decreases only slightly from 9.07 for the fine grid to 9.01 for the coarse grid, indicating a satisfactory grid convergence for the metric of interest herein. It may be noted in passing that a higher grid sensitivity was noted for the amplification characteristics of first mode instabilities along the leeward line of symmetry, which is consistent with the observations in Ref. 17 for the zero out-gassing case.

As described in a previous paper, ${ }^{16}$ two instability mechanisms dominate in the boundary layer flow over this sphere-cone model. First-mode instability is evident along the leeward symmetry plane while crossflow instability prevails elsewhere on the leeward side. Fig. 14 shows the effect of out-gassing on the evolution of crossflow instability modes along selected streamline paths that were used to integrate the amplification rates of stationary and crossflow instabilities along the model surface. The starting point of each streamline indicates the onset of instability along that trajectory. The streamlines stop at the location where a particular $\mathrm{N}$-factor has been reached. These maximum $\mathrm{N}$-factors for stationary and travelling crossflow modes are set to be 5 and 7, respectively. Comparison with the experimentally obtained phosphor thermography image is also included in the figure, and it may be seen that the observed transition fronts correlate well with these preset $\mathrm{N}$-factor values for the baseline (i.e., zero injection) case. The results also highlight the strong effect of out-gassing on the crossflow modes of instability. .The transition fronts have shifted substantially farther upstream as a result of the wallblowing. While not discussed herein, the effect of out-gassing on the first mode instabilities along the leeward line is strongly destabilizing (even more so in comparison with the crossflow modes shown in Fig. 14), analogous to the findings in section IV for the hemi-spherical capsule. It appears that wall blowing introduces additional inflectional points on the velocity profiles, which have a direct impact on the first-mode type instability. On the other hand, the crossflow instability is mainly due to the inflectional instability of the crossflow velocity component, which is not influenced as strongly by the out-gassing.

\section{Conclusions}

Stability computations are carried out for three flow configurations with and without out-gassing in order to obtain initial insights concerning the effects of ablation on the stability of the boundary layer flow over entry/reentry vehicles. For the second mode dominated axisymmetric boundary layer over a hypersonic slender cone, out-gassing actually leads to moderate stabilization of the boundary layer, contrary to the measured trend during a previous wind tunnel experiment. This indicates that the transition in the experiment was caused by instability mechanism other than second mode. On the other hand, the boundary layer flow over the blunt, hemispherical body is dominated by first mode instabilities, which become increasingly more unstable as the magnitude of out-gassing velocity is increased, consistent with an available set of measurements. A similar destabilizing trend is also noted for a blunt sphere-cone model at an angle of incidence which involves crossflow instabilities. Further studies are underway to broaden the parametric range of these cases to provide a deeper understanding of the physical mechanisms underlying these disparate effects on different type of instability modes, including the receptivity and nonlinear stages of the transition process.

\section{Acknowledgments}

This work was performed as part of the Aerodynamics, Aerothermodynamics, and Plasma Dynamics (AAP) discipline of the Hypersonics Project of NASA's Fundamental Aeronautics Program (FAP). Technical discussions with Drs. Baurle, Mazaheri, Thompson, and Marvin at NASA are gratefully appreciated. 


\section{References}

1 Schneider, S., "Laminar-Turbulent Transition on Reentry Capsules and Planetary Probes," J. Spacecraft and Rockets, Vol. 43, No. 6, Nov-Dec. 2006.

2 Chang, C.-L., Choudhari, M., Hollis, B., and Li, F., "Transition Analysis for the Mars Science Laboratory Entry Vehicle," AIAA Paper 2009-4076, 2009.

3 Kaattari, G., "Effects of Mass Addition on Blunt-Body Boundary-Layer Transition and Heat Transfer," NASA TP1139, 1978.

4 Marvin, J. and Akin, C.M., "Combined Effects of Mass Addition and Nose Bluntness on Boundary-Layer Transition," AIAA Journal, Vol. 8, No. 5, May 1970, pp. 857-863.

$5 \quad$ S. Schneider, "Hypersonic Boundary-Layer Transition with Ablation and Blowing," AIAA Paper 2008-3730, 2008.

6 Reda, D., Wilder, W., Bogdonoff, D., and Prabhu, D., "Transition Experiments on Blunt Bodies with Distributed Roughness in Hypersonic Free Flight,” J. of Spacecraft and Rockets, Vol.45, No. 2, pp. 210-215, 2008.

7 Schneider, S.P., "Effects of Roughness on Hypersonic Boundary-Layer Transition," AIAA Paper 2007-305, 2007.

8 Choudhari, M., Li, F., Wu, M., Chang, C.-L., and Edwards, J.A., "Laminar Turbulent Transition Behind Discrete Roughness Elements in a High-Speed Boundary-Layer,” AIAA Paper 2010-1575, 2010.

9 Martinelli, S., Ruffin, S., McDaniel, R., Brown, J., Wright, M., and Hash, D., "Validation Process for Blowing and Transpiration-Cooling in DPLR," AIAA Paper 2007-4255, 2007.

10 Thompson, R.A. and Gnoffo, P., "Implementation of a Blowing Boundary Condition in the LAURA Code," AIAA Paper 2008-1243, 2008.

11 Johnson, H.B., Gronvall, J.E., and Candler, G.V., "Reacting Hypersonic Boundary Layer Stability with Blowing and Suction," AIAA Paper 2009-0938, 2009.

12 Ghaffari, S., Marxen, O., Iaccariono, G., and Shaqfeh, E.S.G., "Numerical Simulations of Hypersonic Boundary-Layer Instability with Wall Blowing," AIAA Paper 2010-306, 2010.

13 Harris, J.E. and Blanchard, D.K., "Computer program for solving laminar, transitional, or turbulent compressible boundary-layer equations for two-dimensional and axisymmetric flow," NASA TM-83207, Feb. 1982.

14 Chang, C.-L., "LASTRAC.3d: Transition Prediction in 3D Boundary Layers," AIAA Paper 2004-2542, 2004.

15 Hollis, B. R., Liechty, D. S., Wright, M. M., Holden, M. S., Wadhams, T. P., MacLean, M., and Dyakonov, A., "Transition Onset and Turbulent Heating Measurements for the Mars Science Laboratory Entry Vehicle," AIAA Paper 2005-1437, 2005.

16 Chang, C.-L., Choudhari, M., Hollis, B.R., and Li, F., "Transition Analysis for the Mars Science Laboratory Entry Vehicle," AIAA Paper 2009-4076, 2009. 


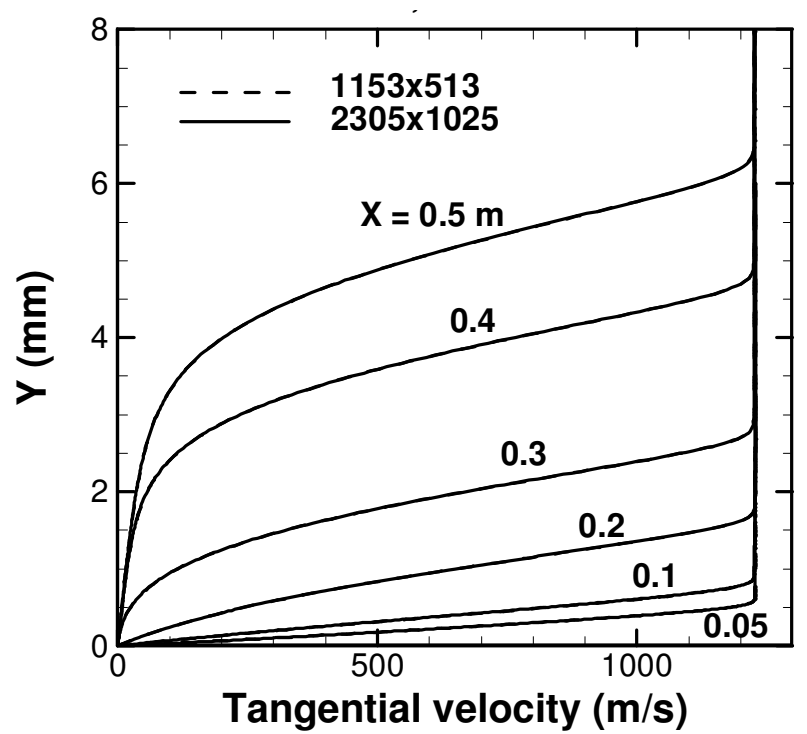

(a) Tangential velocity profiles.



(b) temperature profiles.

Figure 1. Results of grid convergence test for the slender body.

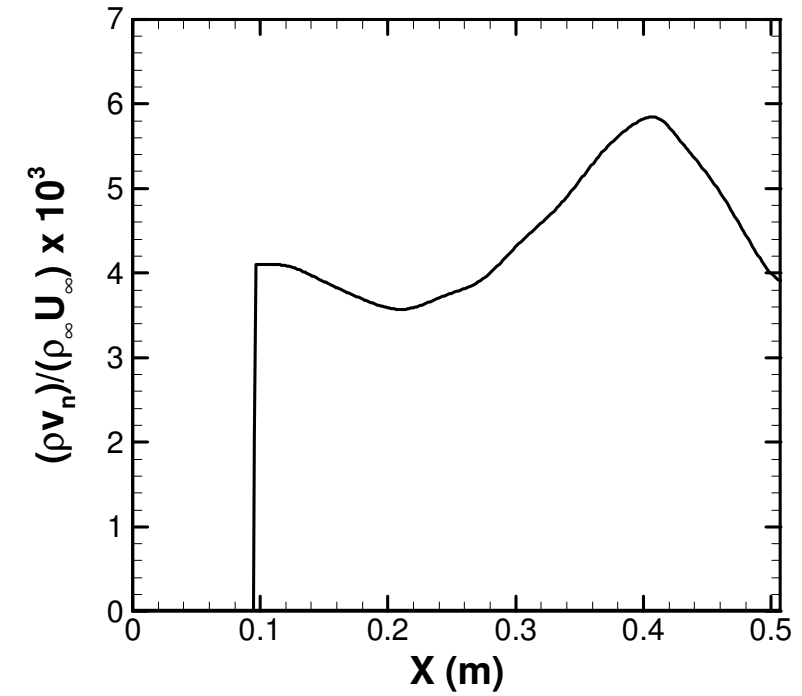

Figure 2. Mass flux distribution over the length of the cone for case II. The mass fluxes for cases III, IV and V are, respectively, 2.61, 4.16 and 9.71 times larger.

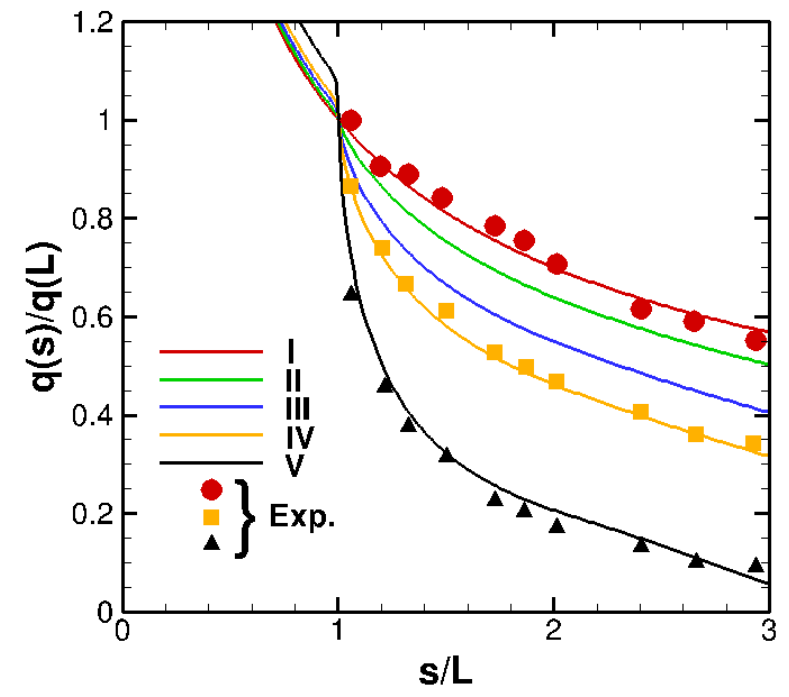

Figure 3. Effect of surface injection on heat transfer over a circular cone. Case I corresponds to impermeable cone (i.e., zero injection), whereas cases II through IV correspond to increasing surface mass flux. Lines (computations), symbols (data $\left.{ }^{4}\right)$. 


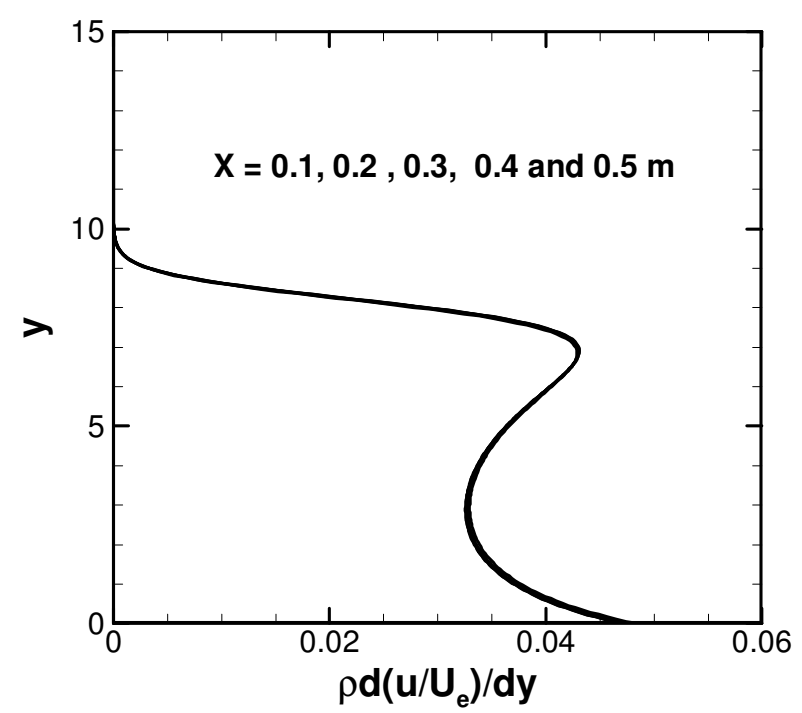

(a) Case I (zero out-gassing). All 5 profiles approximately collapse on to a single curve due to the nearly self-similar nature of the boundary layer in the absence of out-gassing.

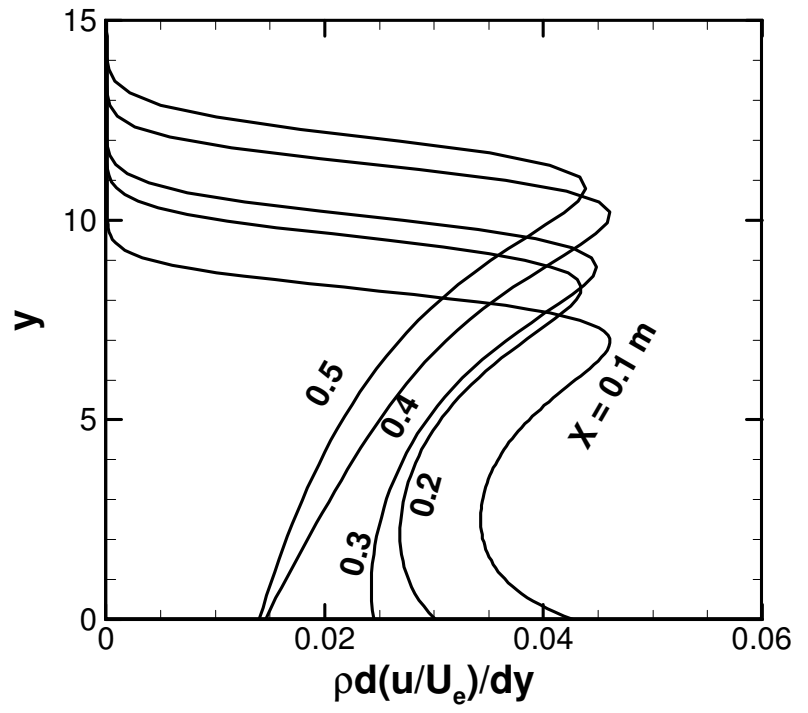

(b) Case IV. Out-gassing mass-flow rate: 4.16 times that of Case II (see Fig.2 above).

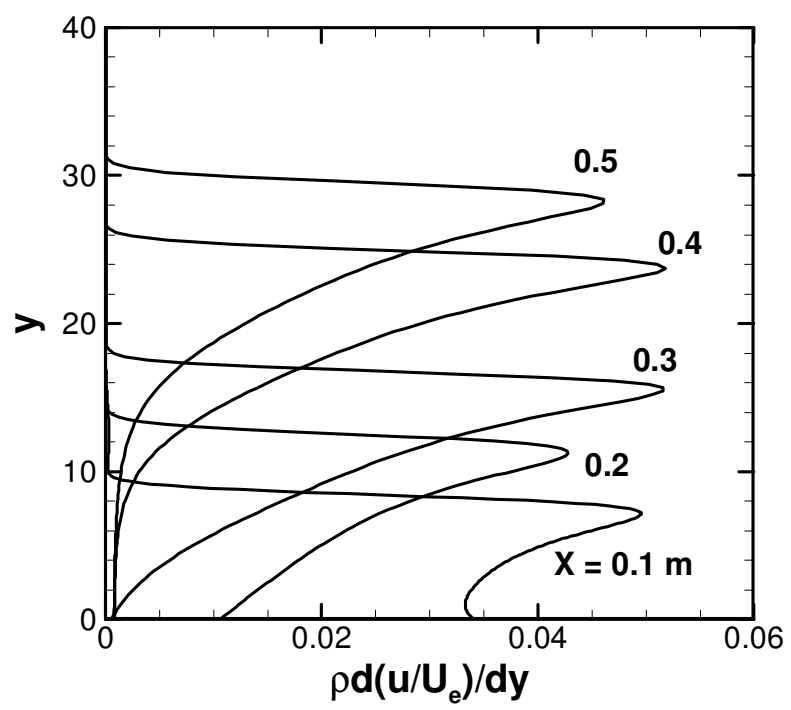

(c) Case V. Out-gassing mass-flow rate: 9.71 times that of Case II (see Fig. 2 above).

Figure 4. $\rho \partial \mathrm{u} / \partial y$ profiles at selected stations along the slender cone. From top to bottom, cases I, IV and $\mathrm{V}$. For each profile, $\rho \partial \mathrm{u} / \partial \mathrm{y}$ start with some finite value at the wall, reaches at peak value in the interior of the flow and decays to zero as $y$ becomes large. Here $y$ is the wall normal distance in selfsimilarity unit (see Nomenclature). 


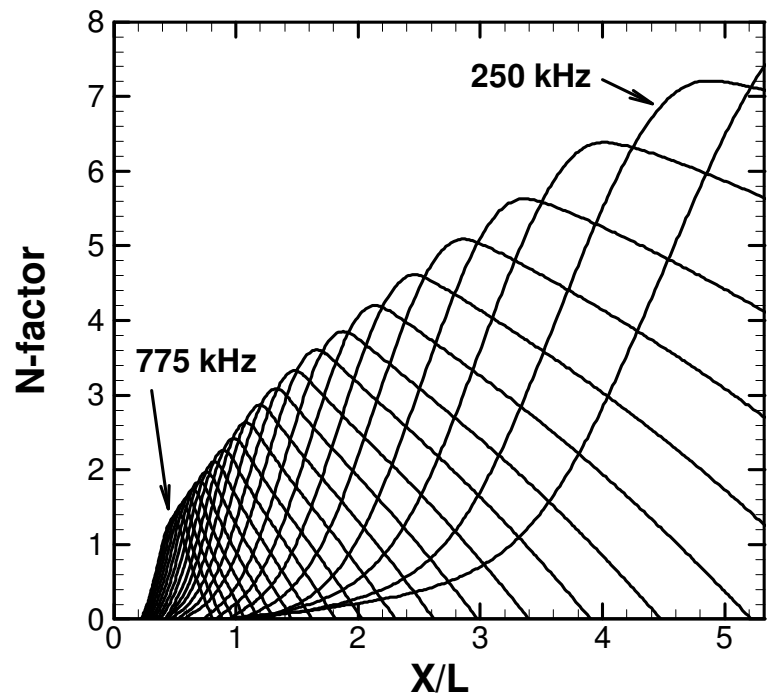

(a) Case I from Fig. 3. Peak frequency is 250 kHz. The $775 \mathrm{kHz}$ curve represents the highest frequency included in computation.

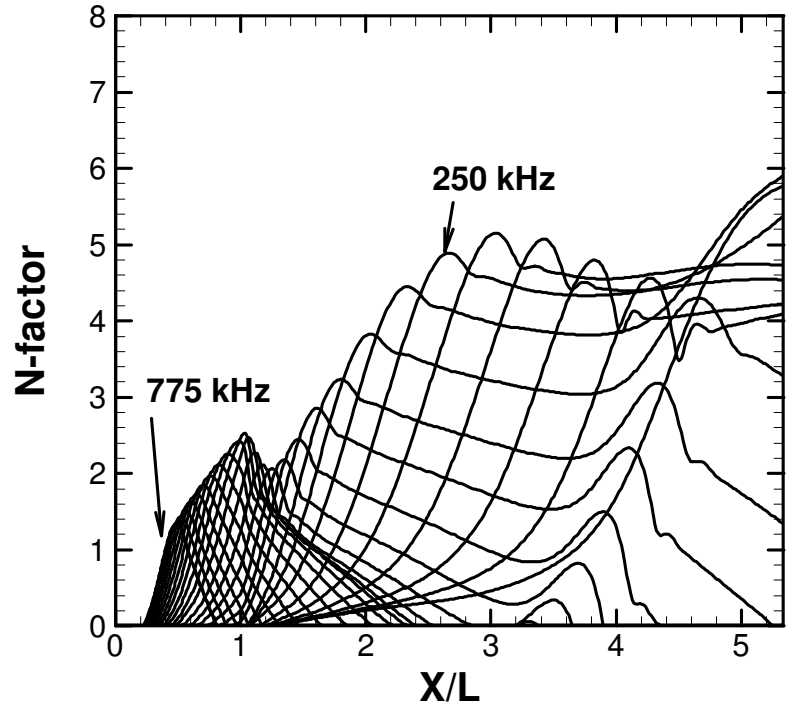

(b) Case IV from Fig. 3. With blowing, the 250 $\mathrm{kHz} \mathbf{N}$-factor curve peaks earlier with lower $\mathrm{N}$ factor than in case $I$.

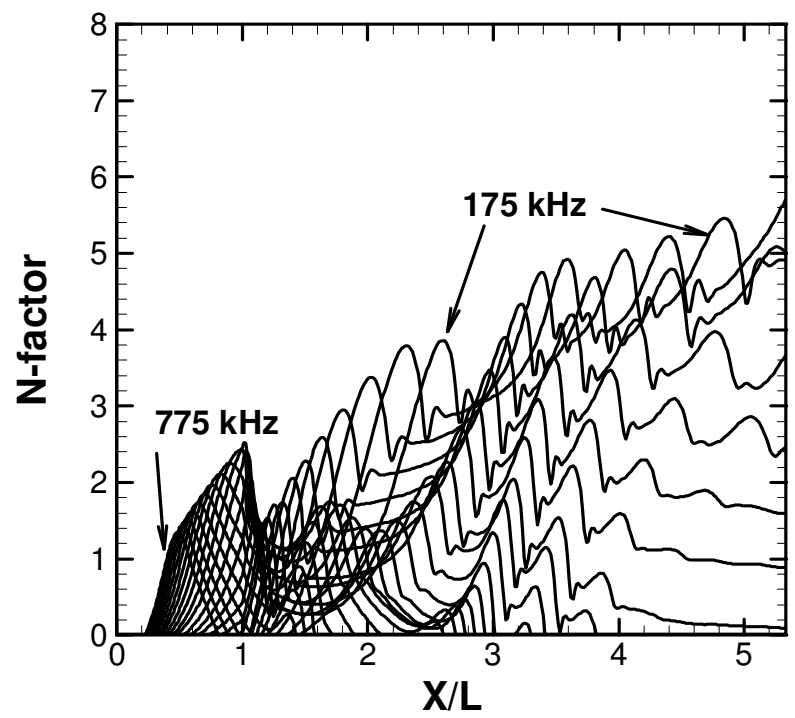

(c) Case V from Fig. 4. Peak frequency lowers due to thickening of boundary layer.

Figure 5. Effect of out-gassing on second mode amplification in cone boundary layer. $\mathrm{N}$-factor evolution for disturbances of selected frequencies. $L$ is the distance from the stagnation point where out-gassing is turned on. The high frequency $\mathrm{N}$-factor curves (e.g. $775 \mathrm{kHz}$ ) amplify mostly ahead of location where blowing starts and, therefore, remain unaffected by out-gassing. 


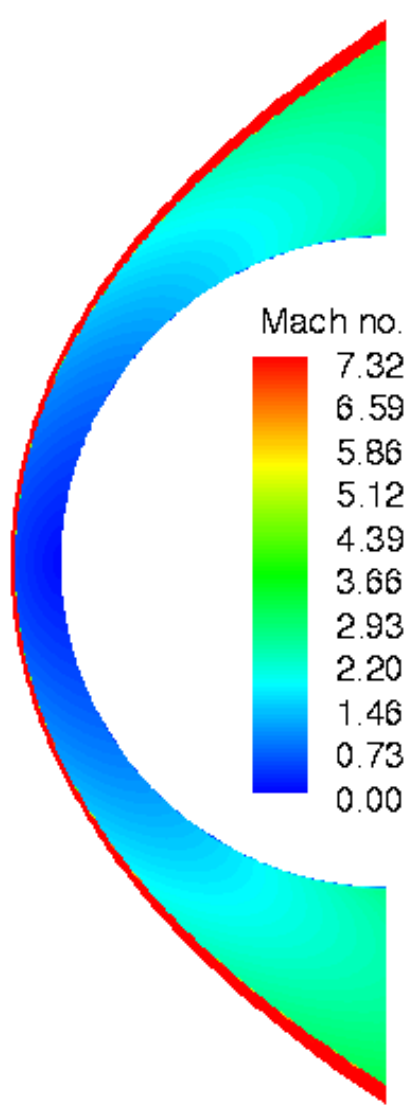

(a) mass flux $=0$

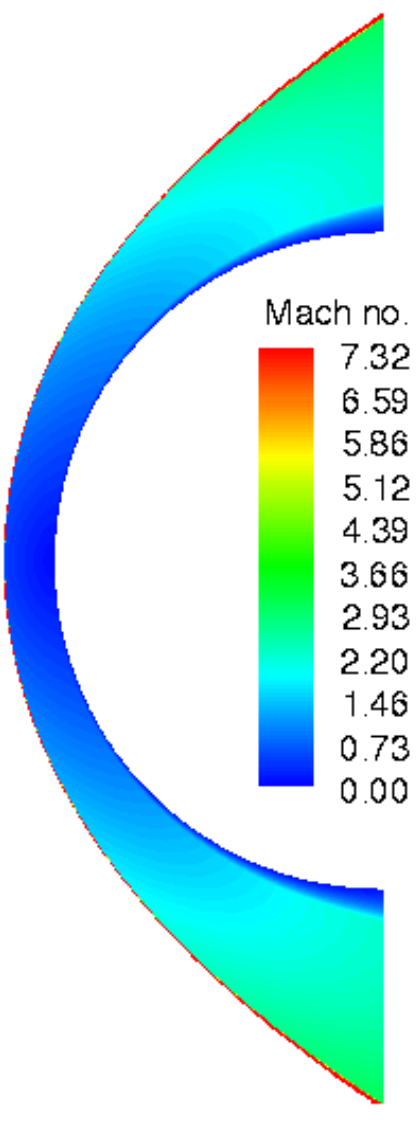

(b) mass flux $=4 \%$

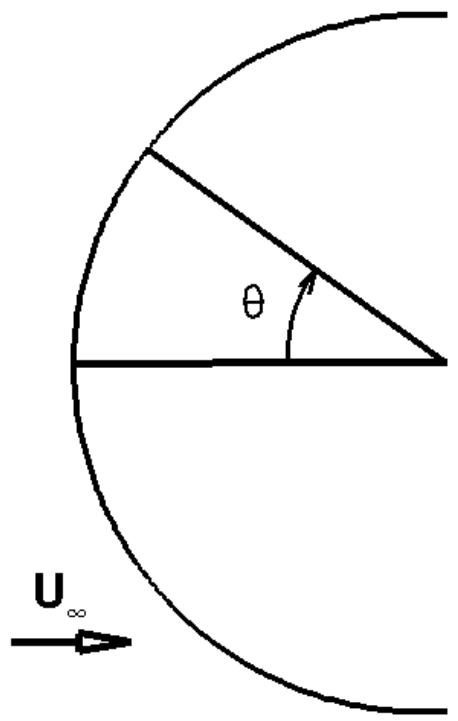

(b) angular coordinate

Figure 6. Mach contours and angular coordinate schematic. (a) No out-gassing; (b) 4\% outgassing, thickening of boundary layer due to out-gassing is obvious in the picture; (c) graphical definition of angular coordinate, $\theta$, referred to in Figs. 7, 8 and 9 below. 


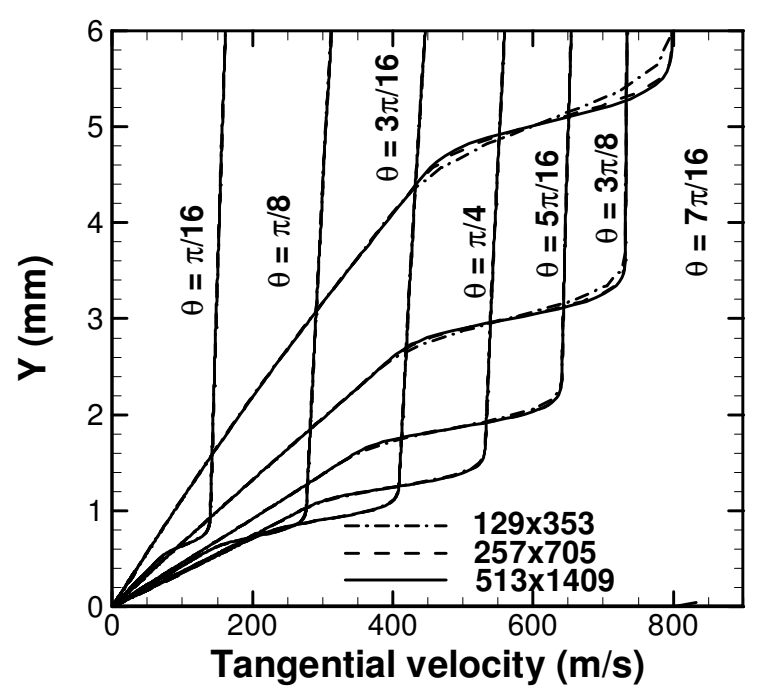

(a)



(b) Line legend, see (a)

Figure 7. Results of grid convergence test for the hemi-spherical body. Injection mass flux is $4 \%$.

The angular coordinate, $\theta$, is explained schematically in Fig. 6 (c) above.



Figure 8. Normalized profile across blunt capsule boundary layer at selected streamwise stations for $\mathbf{m}=\mathbf{0 . 0 1 0}$. The angular coordinate, $\theta$, is explained schematically in Fig. 6 (c) above. The y-coordinate is non-dimensional as given in Nomenclature.



Figure 9. Mode shapes of streamwise velocity perturbation associated with most amplified mode at selected streamwise stations. The angular coordinate, $\theta, \quad$ is explained schematically in Fig. 6 (c) above. The $y$ coordinate is non-dimensional as given in Nomenclature. $\mathbf{m}=\mathbf{0 . 0 1}$. 


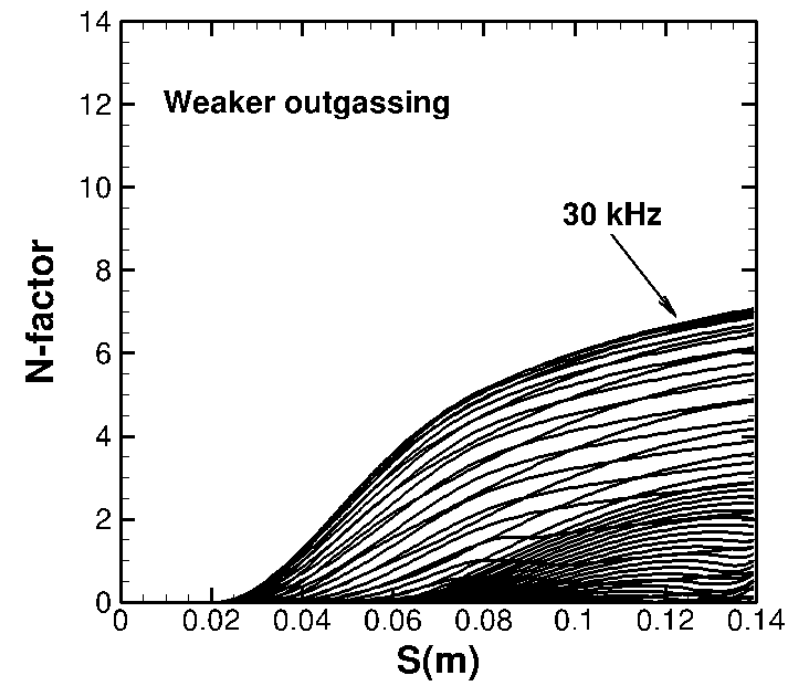

(a) $m=\mathbf{0 . 0 0 1 0}$

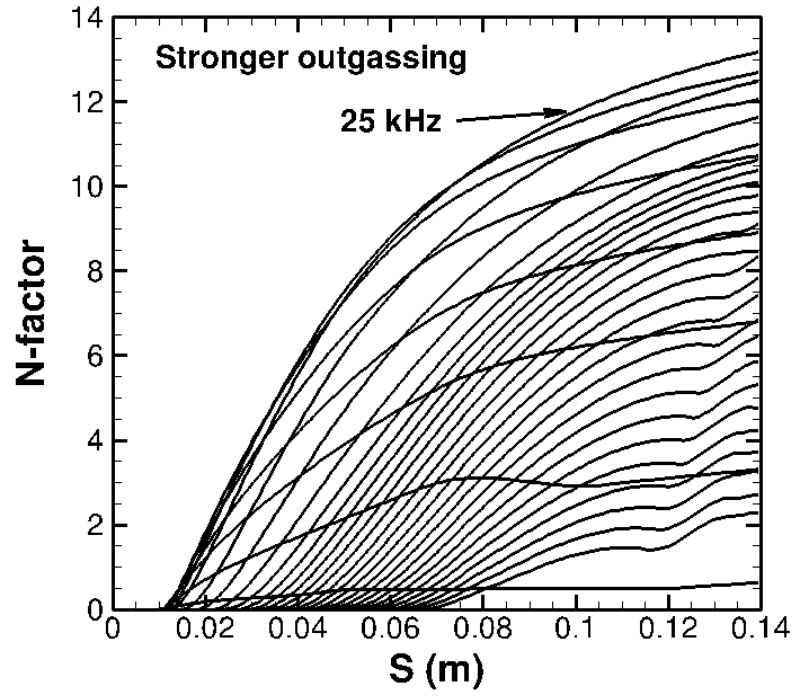

(b) $m=0.0013$

Figure 10. Effect of out-gassing parameter $m$ on instability amplification in boundary layer over a blunt capsule. $\mathrm{N}$-factor curves as a function of distance along the surface for disturbances at selected frequencies. $\mathbf{N}$-factor computations are carried out under quasi-parallel assumption with surface curvature effect accounted for.

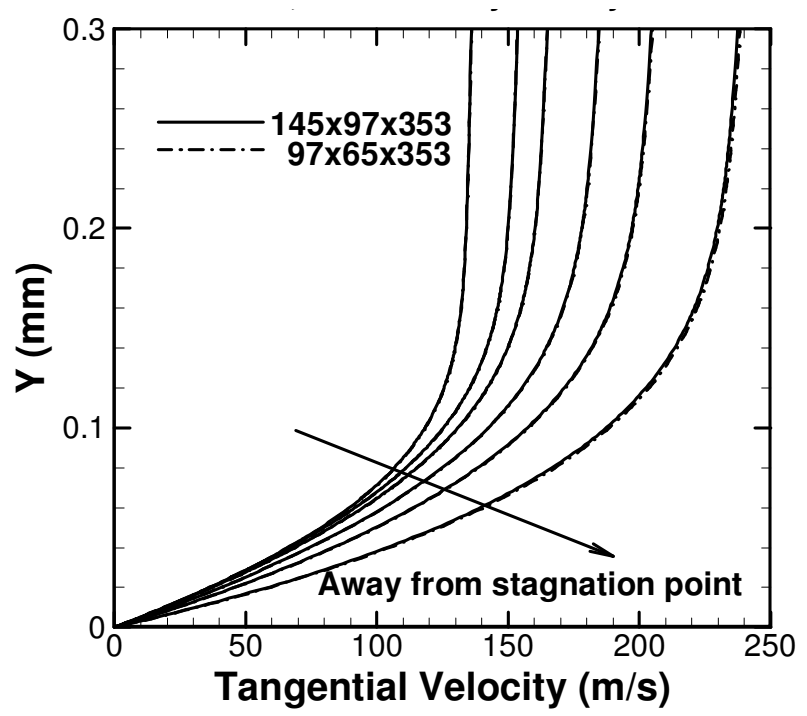

(a) Windward

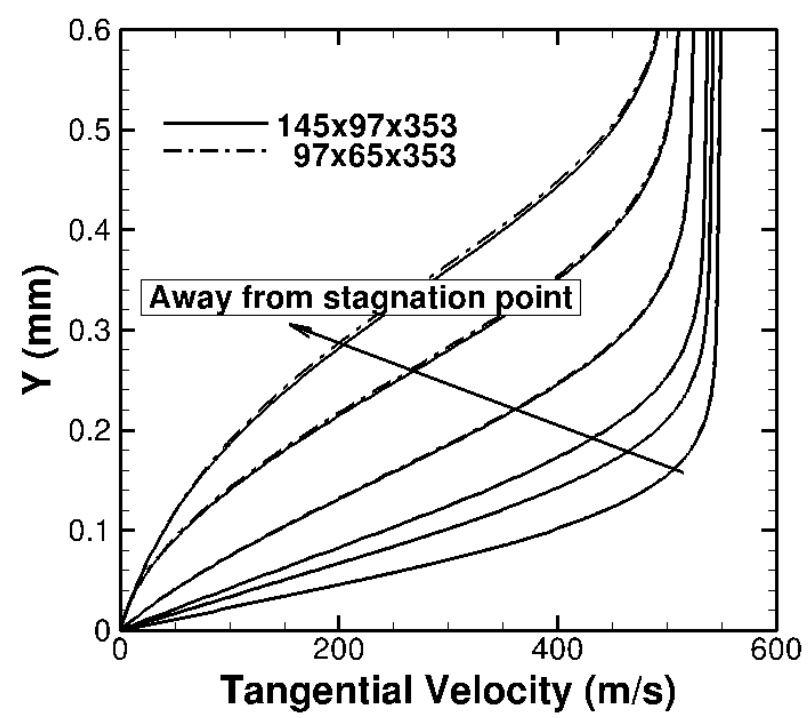

(b) Leeward

Figure11. Velocity profiles along the windward and leeward symmetry lines of MSL model. 


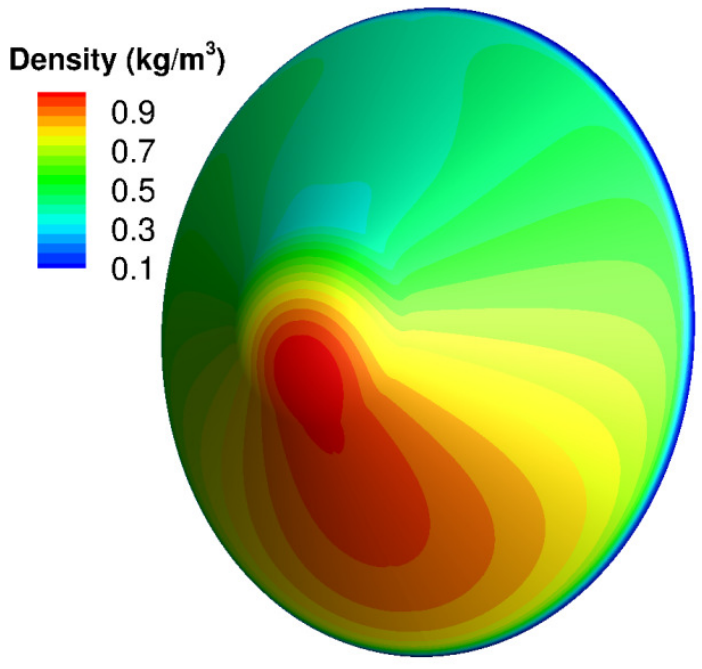

(a) Surface density

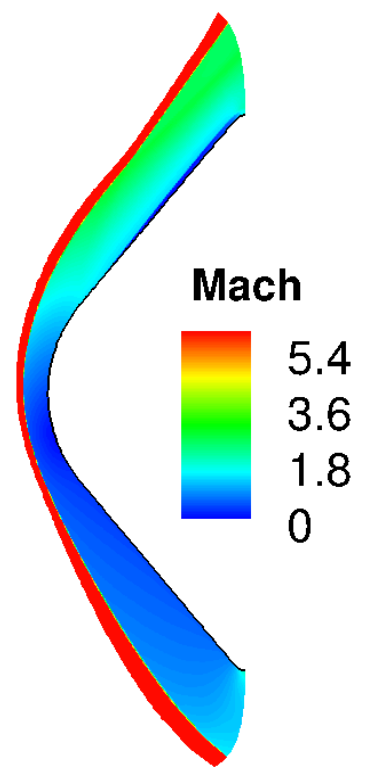

Figure 12. Density and Mach contours of MSL model.

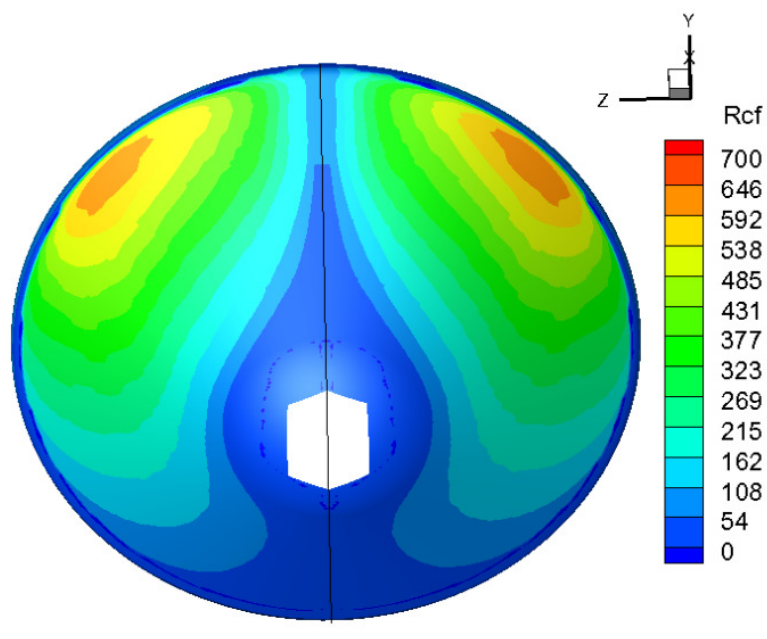

(a) No out-gassing

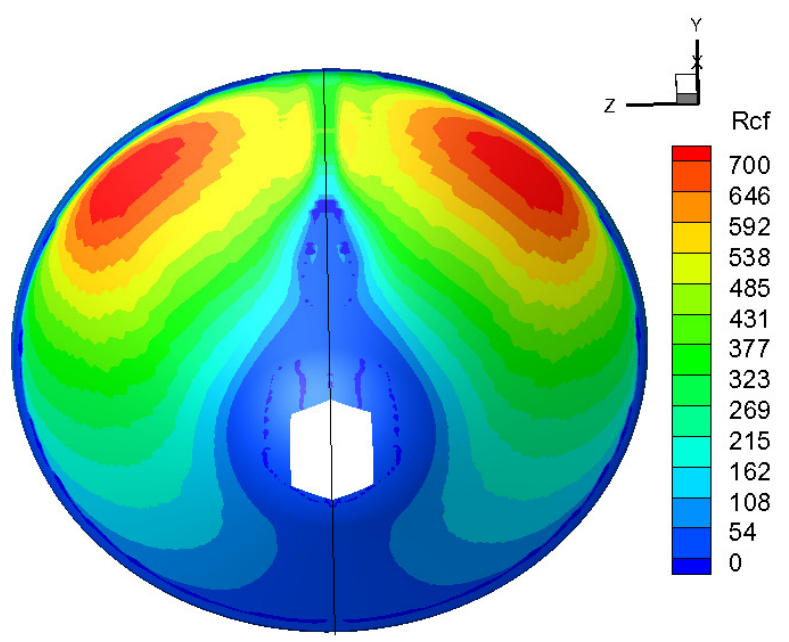

(b) With out-gassing. $m=0.001$

Figure 13. Effect of out-gassing on crossflow Reynolds number $\left(\boldsymbol{R}_{c f}\right)$ distribution on the blunt sphere-cone model (both cases computed with the fine grid tuned to resolve the respective boundary layer flows). 


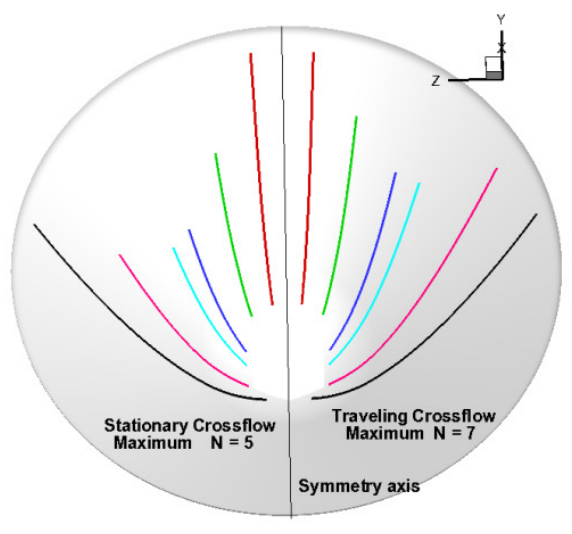

(a) No out-gassing (computational)



(b) No out-gassing (experimental)

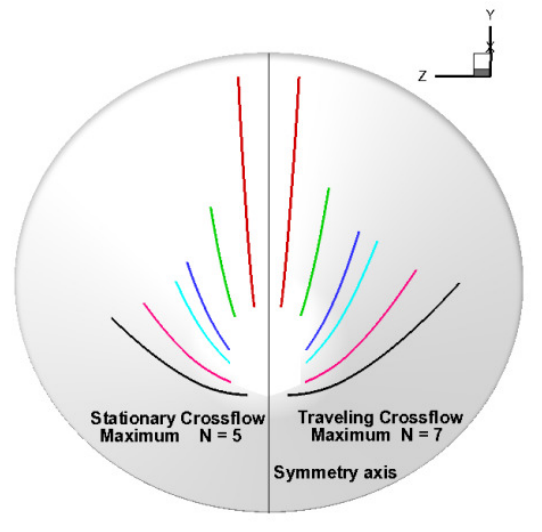

(c) With out-gassing (computational) $m=0.001$

Figure 14. Comparison of predicted and experimental transition fronts using $N=5$ and $N=7$ for stationary and travelling crossflow instability. The asymmetry shown in (b) is most likely due to experimental uncertainties. 